\title{
Hemiplegia and the billowing mitral leaflet syndrome
}

\author{
G. S. H I R S O W I T Z A N D D. S A F F E R \\ From the Departments of Neurology and Medicine, Baragwanath Hospital and the University \\ of the Witwatersrand, Johannesburg, South Africa
}

SUMMARY Four young patients presenting with major neurological episodes and with coexisting prolapsing mitral valve are described. An attempt at correlating the two entities is made. The neurological complications of prolapsing mitral valve are stressed.

Midsystolic clicks and late systolic murmurs heard over the precordium, previously thought to be innocent and extracardiac in origin, are now generally accepted as part of a common and highly variable clinical syndrome caused by a functional abnormality of the mitral valve apparatus. The incidence of prolapsing mitral valve is said to be $0.5 \%$ of the male, and $6 \%$ of the female population (Brown et al., 1975; Procacci et al., 1976). The cardiovascular manifestations have been well documented (Barlow et al., 1963, 1968; Hancock and Cohn, 1966; Barlow and Pocock, 1975; O'Rourke et al., 1975). However, neurological disturbances occurring in association with this disorder have only recently been stressed (Barnett et al., 1976; Malcolm et al., 1976). Four cases of prolapsing mitral valve presenting with major cerebrovascular episodes are described here. Their ages ranged from 16 to 37 years. None showed evidence of atherosclerotic cerebrovascular disease, hypertension, vasculitis, or coagulation defect.

\section{Case reports}

\section{CASE 1}

A 16 year old girl presented in January 1977 with a left hemiplegia of sudden onset. She had fallen down on the day of admission and was unable to walk afterwards. There was no loss of consciousness. She described previous syncopal attacks, occasional palpitations, and shortness of breath, but no chest pain. Examination showed a dense left hemiplegia. She also had absent right brachial and radial pulses. Auscultation revealed an intermittent nonejection click and a late systolic

Address for reprint requests: Dr D. Saffer, Department of Neurology, Baragwanath Hospital, PO Bertsham, 2013, Johannesburg, South Africa.

Accepted 8 November 1977 murmur at the apex. Echocardiography and cardiac cineangiography demonstrated prolapsing mitral valve. Electroencephalography showed no abnormality, while computerised axial tomography (EMI scan) revealed an infarct in the area of the right internal capsule. Postexercise electrocardiography showed occasional ventricular extrasystoles.

\section{CASE 2}

A 33 year old man was admitted to hospital in February 1976 with sudden onset of weakness of the left arm and leg. He gave a history of a previous episode of paraesthesia in the left arm several months before, for which he did not seek medical attention. On examination he was found to have a left hemiplegia involving the arm more than the leg. A nonejection click was heard on auscultation of the heart. A carotid angiogram showed right middle cerebral artery occlusion. Aortic arch angiography was normal, and cardiac cineangiography confirmed the presence of a billowing posterior mitral leaflet. The electrocardiogram was normal.

\section{CASE 3}

A 37 year old woman presented in January 1977 with a right hemiplegia and expressive dysphasia of sudden onset. A transient ischaemic event with similar distribution had occurred three weeks previously. On auscultation of the heart, she was found to have a nonejection click with a late systolic murmur. Echocardiography confirmed the diagnosis of prolapsing mitral valve. A carotid angiogram was normal.

\section{CASE 4}

A 17 year old girl suddenly became paralysed on the left side of the body while at the cinema. On examination she was found to have a left hemiplegia. A carotid angiogram showed occlusion of 
the right middle cerebral artery. Arch angiography was normal. On reassessment 18 months later, a nonejection click with systolic flow murmur was heard. This was confirmed on phonocardiography. There were no electrocardiographic abnormalities. The presence of a billowing mitral valve cusp was confirmed by echocardiography.

\section{Discussion}

Prolapsing mitral valve, although initially described as an asymptomatic and a benign condition, often presents with symptoms of chest pain, dyspnoea, palpitations, dizziness, and syncope. Infective endocarditis with its neurological embolic complications has been reported in mitral leaflet prolapse (Read and Thal, 1966; LeBauer et al., 1967). However, major neurological episodes in the absence of infective endocarditis have only recently been recognised and described. This may take the form of transient ischaemic events and persistent neurological deficit. Malcolm et al. (1976) in a study of 85 patients with prolapsing mitral valve, reported $11(13 \%)$ with major neurological disturbances. Barnett et al. (1976) described 12 cases of prolapsing mitral valve with recurrent transient ischaemic attacks and nonprogressive strokes. Arrhythmias have been well documented in this syndrome (Pocock and Barlow, 1971; Gooch et al., 1972; Sloman et al., 1972; Barlow and Pocock, 1975; Winkle et al., 1975). Although occasionally present in the resting electrocardiogram, arrhythmia is more often induced or aggravated by physical exertion. The increased incidence of sudden death reported by some authors has been attributed to arrhythmia and the overall incidence of sudden death in the literature has been estimated to be $1.2 \%$ (Barlow et al., 1963; Gooch et al., 1972; Marshall and Shappel, 1974; Jeresaty, 1976). The most common disturbance of rhythm is premature ventricular contractions, while supraventricular tachyarrhythmia, atrial premature beats, ventricular tachycardia, and conduction disturbances are encountered frequently.

The syndrome is more prevalent in women, and a high familial occurrence has been found (Pocock and Barlow, 1971). The click, which is usually midsystolic and single though sometimes multiple, may or may not be associated with a murmur of mitral regurgitation. The murmur, typically occurring in late systole, may be confined to early systole, and may even be holosystolic.

The morphology of the mitral valve apparatus and contiguous areas in postmortem and surgically removed specimens has been studied by several authors (Pomerance, 1969; Trent et al., 1970;
Perloff and Roberts, 1972; McKay and Yacoub, 1973; Marshall and Shappel, 1974; Silver, 1974). The deformity has been characterised by the presence of voluminous and redundant thickened valve leaflets with a gelatinous consistency. Microscopically, the affected leaflets show fibrosal degeneration, usually in the central plate, with deposition of a loose metachromatically staining myxomatous tissue containing large amounts of acid mucopolysaccharides. The chordae may be similarly involved. There is marked fibroelastic thickening of the surrounding endocardium. The fibroelastic proliferation is presumably nonspecific and related to the stretching process. Pomerance (1969), in a review of 35 necropsy cases, demonstrated tearing in the endocardium overlying the myxomatous degenerate valve and, in 10 of these cases, he described adherent fibrin and red blood cells in the absence of inflammation. He suggested that the loss of endothelial continuity was secondary to the underlying fibrosal changes and the abrupt variations in tension, an opportune site for fibrin, and red blood cell deposition being created. This "fibrinous endocarditis" may initiate platelet aggregation with the formation of platelet-fibrin mural thrombus. In some cases, left ventricular endocardial fibroelastosis, possibly resulting from friction of the elongated chordae and various haemodynamic stimuli, has been observed (McKay and Yacoub, 1973).

The mode of presentation in our four patients suggests an embolic aetiology. The acuteness of onset, the focal nature of these cerebral ischaemic events, as well as the site of the cerebrovascular occlusion (middle cerebral artery distribution) favour an embolus (L'Hermitte et al., 1968, 1970). All patients were investigated for coagulation defects, collagenosis, diabetes mellitus, syphilitic disease, and atherosclerosis, with negative results. There was no history of ingestion of oral contraceptives. Blood pressures were normal and the most probable origin of the emboli was cardiac. There was no evidence of infective endocarditis. Case 1 showed evidence of multiple emboli involving the right brachial and radial pulses, and two patients had suffered previous ischaemic episodes. Cardiac arrhythmia, manifesting as ventricular ectopic beats, was observed only in case 1 . However, 24 hour cardiac monitoring was not done.

The pathogenesis of the emboli in prolapsing mitral valve is uncertain. It has been suggested that the abnormal mitral valve and perhaps secondary ventricular fibrinous endocardial changes may predispose to thrombus or platelet aggregate formation (Barnett et al., 1976). The presence of paroxysmal cardiac arrhythmia may 
enhance a thrombotic tendency, and would contribute to the process of embolisation. The role of ventricular asynergy is obscure. Mitral regurgitation with left atrial enlargement does not appear to play a significant role in these patients. In view of the possibility of recurrent embolic episodes, the use of anticoagulant therapy and drugs that interfere with platelet function may be indicated.

Unexplained strokes in young people are seen frequently. We feel that the finding of prolapsing mitral valve in these patients is more than coincidental. We wish to emphasise the importance of recognising an association between cerebral ischaemic events and prolapsing mitral valve. This entity should be considered in patients with unexplained strokes, and particularly in young people presenting with the sudden onset of neurological signs. In these patients the billowing mitral leaflet syndrome should be actively excluded.

\section{References}

Barlow, J. B., and Pocock, W. A. (1975). The problem of non-ejection systolic click with associated mitral systolic murmurs: emphasis on the billowing mitral leaflet syndrome. American Heart Journal, 90, 635655.

Barlow, J. B., Pocock, W. A., Marchand, P., and Denny, M. (1963). The significance of late systolic murmurs. American Heart Journal, 66, 443-452.

Barlow, J. B., Bosman, C. K., Pocock, W. A., and Marchand, P. (1968). Late systolic murmurs and nonejection systolic clicks. British Heart Journal, 30, 203-218.

Barnett, H. J. M., Jones, M. W., Boughner, D. R., and Kostuk, W. J. (1976). Cerebral ischemic events associated with prolapsing mitral valve. Archives of Neurology (Chicago), 33, 777-782.

Brown, O. R., Kloster, F. E., and DeMots, H. (1975). The incidence of mitral valve prolapse in the asymptomatic normal. Circulation, 52, 73.

Gooch, A. S., Vicencio, F., Maranhao, V., and Goldberg, H. (1972). Arrhythmia and left ventricular asynergy in the prolapsing mitral leaflet syndrome. American Journal of Cardiology, 29, 611-619.

Hancock, E. W., and Cohn, K. (1966). The syndrome associated with mid-systolic clicks and late systolic murmurs. American Journal of Medicine, 41, 183196.

Jeresaty, R. M. (1976). Sudden death in mitral valve prolapse-click syndromes. American Journal of Cardiology, 37, 317-318.

LeBauer, E. J., Perloff, J. K., and Keliher, T. F. (1967). The isolated systolic click with bacterial endocarditis. American Heart Journal, 73, 534-537.
L'Hermitte, F., Gautier, J. C., and Guiraud, B. (1968). Ischemic accidents in the middle cerebral artery territory: a study of the causes in 122 cases. Archives of Neurology (Chicago), 19, 248-256.

L'Hermitte, F., Gautier, J. C., and Derousne, C. (1970). Nature of occlusion of the middle cerebral artery. Neurology (Minneapolis), 20, 82-88.

Malcolm, A. D., Boughner, D. R., Kostuk, W. J., and Ahuja, S. P. (1976). Clinical features and investigative findings in the presence of mitral leaflet prolapse: study of 85 consecutive patients. British Heart Journal, 38, 244-256.

Marshall, C. E., and Shappel, S. D. (1974). Sudden death and the ballooning posterior leaflet syndrome: detailed anatomical and histochemical investigation. Archives of Pathology, 98, 134-138.

McKay, R., and Yacoub, M. H. (1973). Clinical and pathological findings in patients with floppy valves treated surgically. Circulation, 47/48, 63-72.

O'Rourke, R. A., Crawford, M. H., Johnson, A. D., Davidson, R. M., LcWinter, M. M., and Karliner, J. S. (1975). Prolaping mitral leaflet syndrome. Western Journal of Medicine, 122, 217-231.

Perloff, J. K., and Roberts, W. C. (1972). The mitral valve apparatus: functional anatomy of mitral regurgitation. Circulation, 46, 227-239.

Pocock, W. A., and Barlow, J. B. (1971). Etiology and electrocardiographic features of the billowing posterior mitral leaflet syndrome. American Journal of Medicine, 51, 731-739.

Pomerance, A. (1969). Ballooning deformity (mucoid degeneration) of atrioventricular valves. British Heart Journal, 31, 343-351.

Procacci, P. M., Savran, S. V., Schreiter, S. L., and Bryson, A. L. (1976). Prevalence of clinical mitralvalve prolapse in 1169 young women. New England Journal of Medicine, 294, 1086-1088.

Read, R. C., and Thal, A. P. (1966). Surgical experience with symptomatic myxematous valvular transformation (floppy valve syndrome). Surgery, 59, 173-182.

Silver, M. D. (1974). Rheumatic carditis: acute and chronic atrioventricular valve disease. In The Heart. Edited by J. Edwards, M. Lev, and M. Abel, pp. 87109. Williams and Wilkins: Baltimore.

Sloman, G., Wang, M., and Walker, J. (1972). Arrhythmia and exercise in patients with abnormality of the posterior leaflet of the mitral valve. American Heart Journal, 83, 312-317.

Trent, J. K., Adelman, A. G., Wigle, E. D., and Silver, M. D. (1970). Morphology of a prolonged posterior mitral leaflet. American Heart Journal, 79, 539543.

Winkle, R. A., Lopes, M. G., Fitzgerald, J. W., Goodman, D. J., Schroeder, J. S., and Harrison, D. C. (1975). Arrhythmia in patients with mitral valve prolapse. Circulation, 52, 73. 